\section{IN BRIEF}

\section{$\Rightarrow$ LIVER}

\section{Interfering with acute intermittent porphyria}

An RNA interference therapy (givosiran) targeting ALAS1 reduced the rate of attacks and improved other disease manifestations in patients with acute intermittent porphyria, the most common subtype of acute hepatic porphyria. A total of 94 patients were randomly assigned either givosiran $(n=48)$ or placebo $(n=46)$ in a phase III trial. In 89 patients with acute intermittent porphyria, the mean annualized attack rate was significantly lower in the givosiran group than the placebo group (decreased by $74 \%, P<0.001 ; 3.2$ versus 12.5 ). Other disease manifestations (including pain) also improved in the treatment group. However, a higher frequency of hepatic and renal adverse events was observed in the givosiran group than the placebo group. Givosiran is now approved by the FDA and the European Medicines Agency for adults with acute hepatic porphyria.

ORIGINAL ARTICLE Balwani, M. et al. Phase 3 trial of RNAi therapeutic givosiran for acute intermittent porphyria. N. Engl.J. Med. 382, 2289-2301 (2020)

\section{$\Rightarrow$ GASTROINTESTINAL CANCER}

\section{Ripretinib for advanced gastrointestinal stromal tumours}

A double-blind, randomized, placebo-controlled, phase III trial has shown that ripretinib (a switch-control tyrosine kinase inhibitor) markedly improves progression-free survival in patients with advanced gastrointestinal stromal tumours who were resistant to approved treatments. From across 29 specialized hospitals in 12 locations, 129 patients ( $>18$ years old) were recruited and randomly assigned to receive either oral ripretinib $150 \mathrm{mg}$ once daily $(n=85)$ or placebo once daily $(n=44)$. In the double-blind period, median progression-free survival was 6.3 months $(95 \% \mathrm{Cl} 4.6-6.9)$ in the ripretinib group versus 1.0 month (95\% $\mathrm{Cl} 0.9-1.7)$ in the placebo group (hazard ratio $0.15,95 \% \mathrm{Cl} 0.09-0.25 ; P<0.0001)$. Ripretinib had an acceptable safety profile, with the most common grade 3 or 4 treatment-emergent adverse events including, among others, hypertension and fatigue in the ripretinib group.

ORIGINAL ARTICLE Blay, J.-Y. et al. Ripretinib in patients with advanced gastrointestinal stromal tumours (INVICTUS): a double-blind, randomised, placebo-controlled, phase 3 trial. Lancet Oncol. https://doi.org/10.1016/S1470-2045(20)30168-6 (2020)

\section{$\Rightarrow$ LIVER CANCER}

\section{A combination therapy to keep hepatocellular carcinoma in check}

In patients with unresectable hepatocellular carcinoma (HCC), the combination of atezolizumab (an immune checkpoint inhibitor) and bevacizumab (antibody against VEGF) led to better overall and progression-free survival than sorafenib. The open-label, phase III trial included 336 patients in the atezolizumab-bevacizumab group and 165 patients in the sorafenib group. At the primary analysis, the hazard ratio for death with the combination therapy versus sorafenib was $0.58(95 \% \mathrm{Cl} 0.42-0.79 ; P<0.001)$. Overall survival at 12 months was $67.2 \%$ with atezolizumab-bevacizumab and $54.6 \%$ with sorafenib. Moreover, median progression-free survival was longer in the combination therapy group ( 6.8 months) than the sorafenib group ( 4.3 months). The percentage of grade 3 or 4 adverse events was similar between the treatment groups, although grade 3 or 4 hypertension occurred in $15.2 \%$ of the atezolizumab-bevacizumab group.

ORIGINAL ARTICLE Finn, R. S. et al. Atezolizumab plus bevacizumab in unresectable hepatocellular carcinoma. N. Engl.J. Med. 382, 1894-1905 (2020)
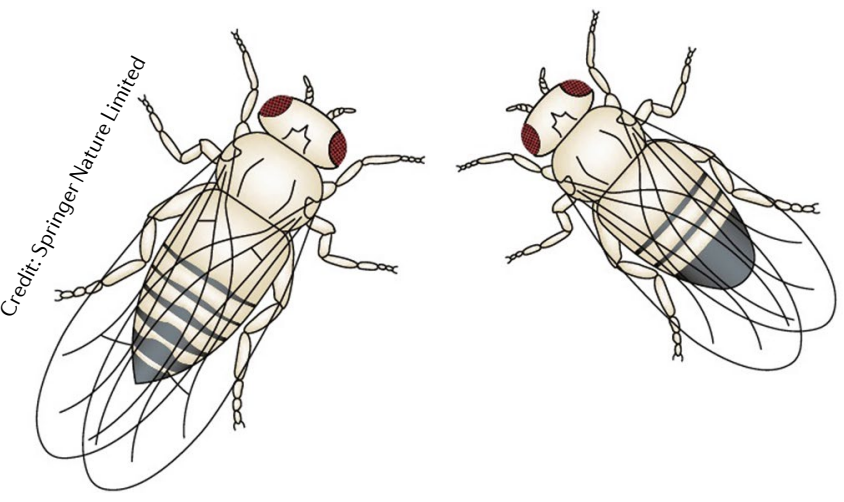

SLEEP

\title{
Sleep loss lethality is caused by gut ROS in mice and flies
}

Severe sleep loss is associated with major health problems, and sleep deprivation can be lethal in model organisms such as rats and flies. But the mechanism underlying this lethality is unknown. In a new study, researchers demonstrate in flies and mice that sleep loss leads to an increase of reactive oxygen species (ROS) in the gut, and that neutralization of ROS can prevent oxidative stress and prolong the lifespan of sleep-deprived flies.

"Sleep ensures proper brain function, so one may guess that sleep deprivation is lethal due to brain damage," says Dragana Rogulja, the corresponding author of the study.

"But the many studies that looked at sleep-deprived brains could never find any major cellular damage." So where in the body is associated with this lethality?

To answer this question, the researchers analysed various tissues for markers of cell damage in flies deprived of sleep using three independent methods. Flies deprived of sleep using thermogenetic stimulation of sleep-suppressing neurons had increased levels of ROS specifically in the gut (mostly the midgut) - when deprivation ceased, ROS levels also gradually decreased. The other sleep deprivation methods corroborated this observation. In addition, the guts of sleep-deprived flies displayed evidence of oxidative stress (which can cause cell death) after the accumulation of ROS.

To confirm these findings in mammalian models, C57BL/6 J and $\mathrm{CBA} / \mathrm{CaJ}$ mice were deprived of sleep using mechanical stimulation. ROS

levels in the small and large intestines of sleep-deprived mice were higher than in non-deprived controls, as were markers of DNA damage and cell death.

Finally, the researchers assessed whether ROS neutralization using food supplementation could extend the survival of sleep-deprived flies. Out of a panel of 53 compounds with antioxidant properties, 11 were able to clear ROS and rescue survival without increasing sleep, and supplementation with any of 4 of these compounds (melatonin, lipoic acid, NAD and sodium phenylbutyrate) had no effect on survival of non-deprived flies. To confirm the role of the gut, transgenic expression of antioxidant enzymes in the gut prevented oxidative damage and extended the survival of sleep-deprived flies. "Animals can be sleepless and have normal lifespan, whereas they would otherwise die prematurely," says Rogulja.

Together, the findings posit a relationship between ROS-induced oxidative damage and the lethality of severe sleep deprivation. Is this work relevant for humans? "We think it is, as we also studied milder deprivation conditions, and they can still lead to ROS accumulation, just slower," notes Rogulja. "Our ultimate goal is to develop therapies that would offset health damage caused by poor sleep."

Jordan Hindson

ORIGINAL ARTICLE Vaccaro, A. et al. Sleep loss can cause death through accumulation of reactive oxygen species in the gut. Cell 181, 1307-1328 (2020) 